Pacific Journal of Mathematics

COMPLETELY DECOMPOSABLE GROUPS WHICH ADMIn 


\title{
COMPLETELY DECOMPOSABLE GROUPS WHICH ADMIT ONLY NILPOTENT MULTIPLICATIONS
}

\author{
C. Vinsonhaler and W. J. Wickless
}

A triangle of size $n$ is a collection $\left\{A_{u}\right\}$ of $n(n+1) / 2$ (not necessarily distinct) rank one torsion-free abelian groups indexed by all integer sequences of the form $u=i, i+1, \cdots$, $i+j$ with $1 \leqq i \leqq i+j \leqq n$, satisfying $T\left(A_{u}\right)+T\left(A_{s}\right) \leqq T\left(A_{u s}\right)$ for all consecutive sequences $u, s$. Here $T\left(A_{v}\right)$ denotes the type of the rank one torsion-free abelian group $A_{v}$. If $A=$ $\oplus_{i \in I} A_{\imath}$ is a direct sum of rank one torsion-free abelian groups $A_{i}$, let $\Delta(A)=\sup \{n \mid \exists$ a triangle of size $n$ of groups chosen, possibly with repetitions, from $\left.\left\{A_{i} \mid i \in I\right\}\right\}, \Delta^{\prime}(A)=\sup \{n \mid \exists$ a triangle of size $n$ of groups chosen without repetition from $\left.\left\{A_{i} \mid i \in I\right\}\right\}$. An abelian group $(G,+)$ is radical iff whenever $(R,+, \cdot)$ is a ring with $(R,+) \cong(G,+)$ there exists a positive integer $n$ with $R^{n}=(0)$.

Theorem. Let $A=\bigoplus_{i \in I} A_{i}$ be such that $\left\{T\left(A_{2}\right) \mid i \in I\right\}$ is an ordered set and $\Delta(A)<\infty$. Then $A$ is radical.

Theorem. Let $A=\oplus_{\imath \in I} A_{i}$ be such that $\Delta^{\prime}(A)=\infty$. Then $A$ is not radical.

Theorem. Let $A=\oplus_{i \in I} A_{i}, B=\oplus_{j \in J} B_{j}$ be such that $\Delta(A)<\infty, \Delta(B)<\infty$. Then if $\left\{T\left(A_{i}\right) \mid i \in I\right\} \cup\left\{T\left(B_{j}\right) \mid j \in J\right\}$ is an ordered set $A \oplus B$ is radical. $A$ bound is given for the index of nilpotency of any multiplication on $A \oplus B$.

1. Preliminaries. Several authors ([2], [3], [4], [5]) have studied the class of abelian groups $(A,+)$ which admit only a trivial ring structure; i.e., if $(R,+, \cdot)$ is a ring with $(R,+) \cong(A,+)$, then $R^{2}=(0)$. These are called nil groups.

In [6] a larger class was introduced-abelian groups which admit only nilpotent multiplications. More precisely:

DEFINITION 1.1. An abelian group $(A,+)$ is a radical group iff whenever $(R,+, \cdot)$ is a ring with $(R,+) \cong(A,+)$, we have $R^{n}=0$ for some positive integer $n$.

In [6], using the techniques of [1], the class of finite rank torsion free radical groups was studied, and it was shown that this class is closed under finite direct sums.

In this paper we study completely decomposable radical groups. We work toward the goals of characterizing such groups and of obtaining information on finite direct sums of such groups.

Throughout, the word "group" means torsion-free abelian group. We let $A=\bigoplus_{i \in I} A_{i}, B=\bigoplus_{j \in J} B_{j}$ be arbitrary completely decompo- 
sable groups; i.e., $I, J$ are arbitrary index sets, $\operatorname{rank} A_{i}=\operatorname{rank} B_{j}=1$ for all $i \in I, j \in J$.

The standard notions of height and type for torsion-free groups ([2], § 42) are used. If $g \in G$, then $h_{G}(g), T_{G}(g)$ denote the height vector and type respectively of $g$ in $G$. The subscript is deleted when no confusion will result. Sums of height vectors and sums of types are defined in the obvious way. If rank $G=1$, the type of $G, T(G)$, is $T(g)$ where $0 \neq g \in G$. A type is called nil iff it is of the form $\left[\left\langle m_{k}\right\rangle\right]$, where $0<m_{k}<\infty$ for infinitely many $k$. ([ $\left.\left\langle m_{k}\right\rangle\right]$ denotes the equivalence class of the height vector $\left\langle m_{k}\right\rangle$.)

\section{Triangles of rank one groups.}

Definition 2.1. A triangle of size $n$ is a collection of groups of rank 1 indexed by all sequences of the form $(i, i+1, i+2, \cdots$, $i+j)$ where $1 \leqq i \leqq n, 0 \leqq j \leqq n-i$, such that $T\left[A_{(i, i+1, \cdots, i+j)}\right]+$ $T\left[A_{(i+j+1, i+j+2, \cdots, i+j+k)}\right] \leqq T\left[A_{(i, i+1, \cdots, i+j+k)}\right]$ for all $i, j$ as above and $k$ with $i+j+k \leqq n$.

It is easiest to consider these triangles in terms of diagrams as in the following.

EXAMPLE. $n=3$.

A triangle of size 3 looks like $A_{1} \quad A_{2} \quad A_{3}$ $A_{12} A_{23}$

with $T\left(A_{1}\right)+T\left(A_{2}\right) \leqq T\left(A_{12}\right)$

$$
\begin{aligned}
& T\left(A_{2}\right)+T\left(A_{3}\right) \leqq T\left(A_{23}\right) \\
& T\left(A_{1}\right)+T\left(A_{23}\right) \leqq T\left(A_{123}\right) \\
& T\left(A_{12}\right)+T\left(A_{3}\right) \leqq T\left(A_{123}\right) .
\end{aligned}
$$

Now let $A=\bigoplus_{i \in I} A_{i}$. Clearly, if $T\left(A_{i}\right)$ is non-nil for some $i$, then $A$ is not radical ([2]). Since we are interested in completely decomposable radical groups, we assume in what follows that $T\left(A_{i}\right)$ is nil for all $i$.

It is well known [3] that such an $A$ is a nil group iff, for all $i, j, k \in I, T\left(A_{i}\right)+T\left(A_{j}\right) \geqq T\left(A_{k}\right)$ - in other words no triangle of size 2 can be formed using $\left\{A_{i} \mid i \in I\right\}$. The following theorems generalize this result by relating multiplications on $A$ which yield nonzero products of length $n$ to triangles of size $n$.

THEOREM 2.1. Let $\left\{A_{i} \mid i \in I\right\}$ be a collection of rank one groups such that $\left\{T\left(A_{i}\right) \mid i \in I\right\}$ is an ordered set. Let * be a multiplication associative on $A=\bigoplus_{i \in I} A_{i}$ with $x_{1}{ }^{*} x_{2}{ }^{*} \ldots{ }^{*} x_{n} \neq 0$ for some 
$\left\{x_{1}, \cdots, x_{n}\right\} \subseteq A$. Then a triangle of size $n$ can be formed by choosing (possibly with repetitions) groups from $\left\{A_{i} \mid i \in I\right\}$.

Proof. Let $x_{1}{ }^{*} x_{2}{ }^{*} \ldots{ }^{*} x_{n} \neq 0$ and choose, for each $i$, some $0 \neq$ $a_{i} \in A_{i}$. Write each $x_{j}$ as a finite rational combination $x_{j}=\sum_{\text {finite }} \alpha_{j i} a_{i}$. It follows immediately that $a_{i_{1}}{ }^{*} a_{i_{2}}{ }^{*} \ldots{ }^{*} a_{i_{n}} \neq 0$ for some (not necessarily distinct) indices $\left\{i, \cdots, i_{n}\right\} \subseteq I$. Reindex, setting $A_{1}=A_{i_{1}}$, $A_{2}=A_{i_{2}}, \cdots, A_{n}=A_{i_{n}}$. The groups $A_{1}, \cdots, A_{n}$ form the top row of the triangle.

For $1 \leqq i<i+j \leqq n$ write $a_{i}{ }^{*} \ldots * a_{i+j}=\Sigma \alpha_{s} a_{s}$, and let $A_{i \cdots i+j}$ by any $A_{s}$ such that $T\left(A_{s}\right)=T_{A}\left(a_{i}{ }^{*} \ldots{ }^{*} a_{i+j}\right)$. Such $A_{s}$ always exists since $\left\{T\left(A_{i}\right) \mid i \in I\right\}$ is an ordered set. It is easy to check that the types of the rank one groups thus chosen satisfy the additive requirements of Definition 2.1.

The requirement that $\left\{T\left(A_{i}\right) \mid i \in I\right\}$ be an ordered set cannot be deleted in the above theorem. One can construct a completely decomposable group $A=\bigoplus_{i=1}^{11} A_{i}$ such that no triangle of size 4 can be formed by choosing groups from $\left\{A_{i} \mid 1 \leqq i \leqq 11\right\}$ but such that $A^{4} \neq(0)$ under an appropriate multiplication. (The numbers 11 and 4 are the minimum rank and index for such an example.)

THEOREM 2.2. Let $\left\{A_{i} \mid i \in I\right\}$ be a set of rank one groups such that a triangle of size $n$ can be formed by choosing $n(n+1) / 2$ distinct groups from the set. Then there is a multiplication on $A=\bigoplus_{i \in I} A_{i}$ with a nonzero product of length $n$.

Proof. Reindex the groups chosen from $\left\{A_{i} \mid i \in I\right\}$ as in Definition 2.1. For each index, $u$, choose $0 \neq a_{u} \in A_{u}$ such that for consecutive indices $r=i \cdots i+j, s=i+j+1 \cdots i+j+k, i+j+k \leqq n, h\left(a_{r}\right)+$ $h\left(a_{s}\right) \leqq h\left(a_{i \cdots i+j+k}\right)=h\left(a_{r s}\right)$. Such a choice is possible because of the type relationships in the triangle. Now define $a_{r} \cdot a_{s}=a_{r s}$ for all consecutive indices $r, s$. If $r$ and $s$ are not of the above form, define $a_{r} \cdot a_{s}=0$. If $A_{i}$ is not a group in the triangle, let $A_{i} A=A A_{i}=(0)$. These products define a unique associative ring structure on $A$. Moreover, in this ring $a_{1} \cdot a_{2} \cdots a_{n} \neq 0$.

Note that the above method (or simple variations of it) cannot be used to obtain multiplication on $\bigoplus A_{i}$ from triangles formed by groups arbitrarily chosen from $\left\{A_{i} \mid i \in I\right\}$ If the same group is allowed in repeated positions, it seems impossible to overcome the consistency problems arising in the definitions of the products $a_{r} \cdot a_{s}$. However, we can obtain a nonassociative multiplication in this case.

The results of this section can be used to obtain information on completely decomposable radical groups and on direct sums of such 
groups. First we prove theorem on the existence of subtriangles of a given triangle.

\section{Subtriangles.}

Definition 3.1. Let $\left\{A_{u}\right\}$ be a triangle of size $n$, indexed as in Definition 2.1. For $1 \leqq m \leqq n$, a subtriangle of size $m$ is an array of $m(m+1) / 2$ (not necessarily distinct) groups chosen from $\left\{A_{u}\right\}$ such that the resulting array, after reindexing, satisfies the requirements of Definition 2.1.

ExAMPle. The array $A_{1} \quad A_{34} \quad A_{5}$ is a subtriangle of any

$$
A_{1234} A_{345}
$$

triangle of size $n \geqq 6$.

Theorem 3.1. Let $A=\left\{A_{i}\right\}, B=\left\{B_{i}\right\}$ and let $n$ be any positive integer. If $m$ is any integer such that $m \geqq f(n) \equiv \max \{3 n-1$, $n(n+1) / 2\}$, then any triangle of size $m^{m}$ of groups from $A \cup B$ contains a subtriangle of size $n$ of groups from $A$ alone or from $B$ alone.

Proof. Use induction on $n$. The result is easy for $n=1$. Now consider a triangle of size $m^{m}$ of groups from $A \cup B$, when $m \geqq f(n)$, ( $n$ an integer not less than 2). As in Diagram 1, divide the first $m^{m-1}$ rows into $m$ disjoint subtriangles of size $m^{m-1}$. Since $m^{m-1} \geqq$

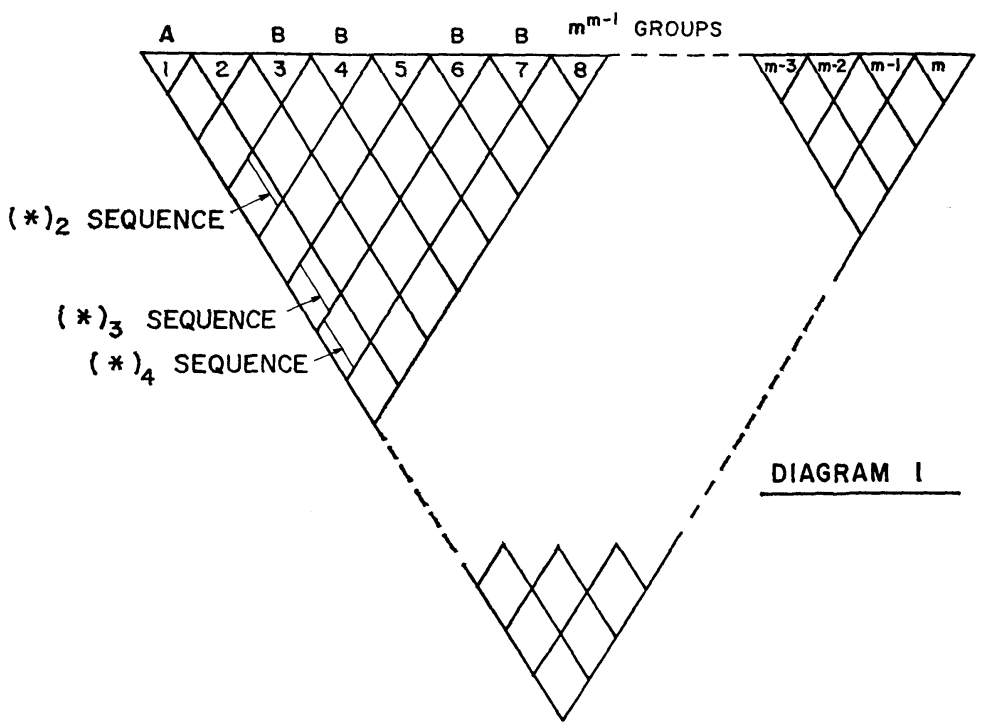


$(m-1)^{m-1}$ and $m-1 \geqq f(n-1)=\max \{3(n-1)-1,(n-1) n / 2\}$, by the induction hypothesis, each of these disjoint triangle of size $m^{m-1}$ contains a subtriangle of size $n-1$ of groups from $A$ alone or from $B$ alone.

Case I. Both triangles of size $n-1$ of groups from $A$ and triangles of size $n-1$ of groups from $B$ appear as subtriangles of the triangles of size $m^{m-1}$. In this case, there occurs a subtriangle of size $n-1$ of groups from one collection, say $A$, such that either to the right or to the left of this subtringle there are $n$ disjoint triangles of size $m^{m-1}$, each of which contains subtriangles of size $n-1$ of groups from $B$. This is due to the fact that in any arrangement of $3 n-1$ objects which are of two types, one of the types must have $n$ objects of the other type either to the right or to the left.

Without loss of generality, assume that the left-most triangle (1) contains a subtriangle of size $n-1$ of groups from $A$, and that there are $n$ triangles to the right which contain subtriangles of groups from $B$. The technique is to expand one of these subtriangles to a triangle of size $n$.

Let $G_{i_{k}, i_{k}+1, \cdots, i_{k}+j_{k}}$ be the rightmost group in the $k^{\text {th }}$ row of the subtriangle of groups from $A$ contained in triangle $1,1 \leqq k \leqq n-1$ (see Diagram 1). If triangle $r_{k}, 1 \leqq k \leqq n$, contains the $k$ th subtriangle to the right of triangle 1 of groups from $B$, consider, for all $2 \leqq$ $k \leqq n$, the sequence of groups

$(*)_{k}$

$$
\left.G_{i_{k}, i_{k}+1, \cdots,\left(r_{k},-1\right) m m-1+1} G_{i_{k}, i_{k}+1}, \cdots,\left(r_{k}-1\right)^{m-1}+2\right), \ldots, G_{i_{k}, i_{k}+1, \cdots, r_{k} m^{m-1}} .
$$

(Each of these sequences is represented by a diagonal in Diagram 1.)

Claim: Either $(*)_{k}$ contains a group from $A$ or the subtriangle of groups from $B$ in triangle $r_{k}$ can be expanded to a triangle of size $n$ of groups from $B$. Suppose $(*)_{k}$ contains no group from $A$. Let $B^{1}$ be any group from $B$ in triangle $r_{k-1}$. Now let $B^{l}, 2 \leqq l \leqq n$, be the group in the sequence $(*)_{k}$ whose index ends in the same number as the index of the left-most group in the $l-1$ row of the subtriangle of groups from $B$ in triangle $r_{k}$. By adjoining each $B^{l}$ as the left-most group in the $l$ th row of the triangle, the subtriangle of groups from $B$ in triangle $r_{k}$ is expanded to a triangle of size $n$ of groups from $B$. In this case we are done, so assume now that each sequence $(*)_{k} 2 \leqq$ $k \leqq n$, contains a group from $A$-call it $A^{k}$. Let $A^{1}$ be any group from $A$ in triangle $r_{1}$ (if none exists, we're done). Now by adjoining $A^{k}$ as the right-most group in the kth row of the $A$-subtriangle of triangle 1 we obtain an $A$-subtriangle of size $n$.

Case II. There exist only $A$-subtriangles of size $n-1$ in the triangles $1,2, \cdots, m$. If this is the case, then any subtriangle of 
size $m^{m-1}$ contained in the first $m^{m-1}$ rows most contain only $A$ subtriangles of size $n-1$. If there were a $B$-subtriangle of size $n-1$, the techniques of Case I could be applied to construct an $A$ or $B$-subtriangle of size $n$.

Now consider any diagonal sequence of the form $(+): G_{i, \ldots, i+j}$, $G_{i, \cdots, i+j+1}, \cdots, G_{i, \cdots, i+j+m^{m-1}-1}$ where $2 \leqq i \leqq m^{m}-m^{m-1}-n-1, n \leqq$ $j \leqq m^{m}-m^{m-1}-i$. These $(+)$ sequences are illustrated in Diagram 2 . If all of the groups in ( + ) are in $A$ then consider the $A$-subtriangle of size $n-1$ contained in the triangle of size $m^{m-1}$ whose top row is $G_{i \div j}, G_{i+j+1}, \cdots, G_{i+j+m^{m-1}-1}$. (Refer to Diagram 2.) Again using the techniques of Case I, this $A$-subtringle can be expanded to a triangle of size $n$ using the sequence $(t)$. Therefore, without loss of generality, assume that every sequence $(+)$ contains a group from $B$. This assumption can be used to build a $B$-subtriangle of size $n$.

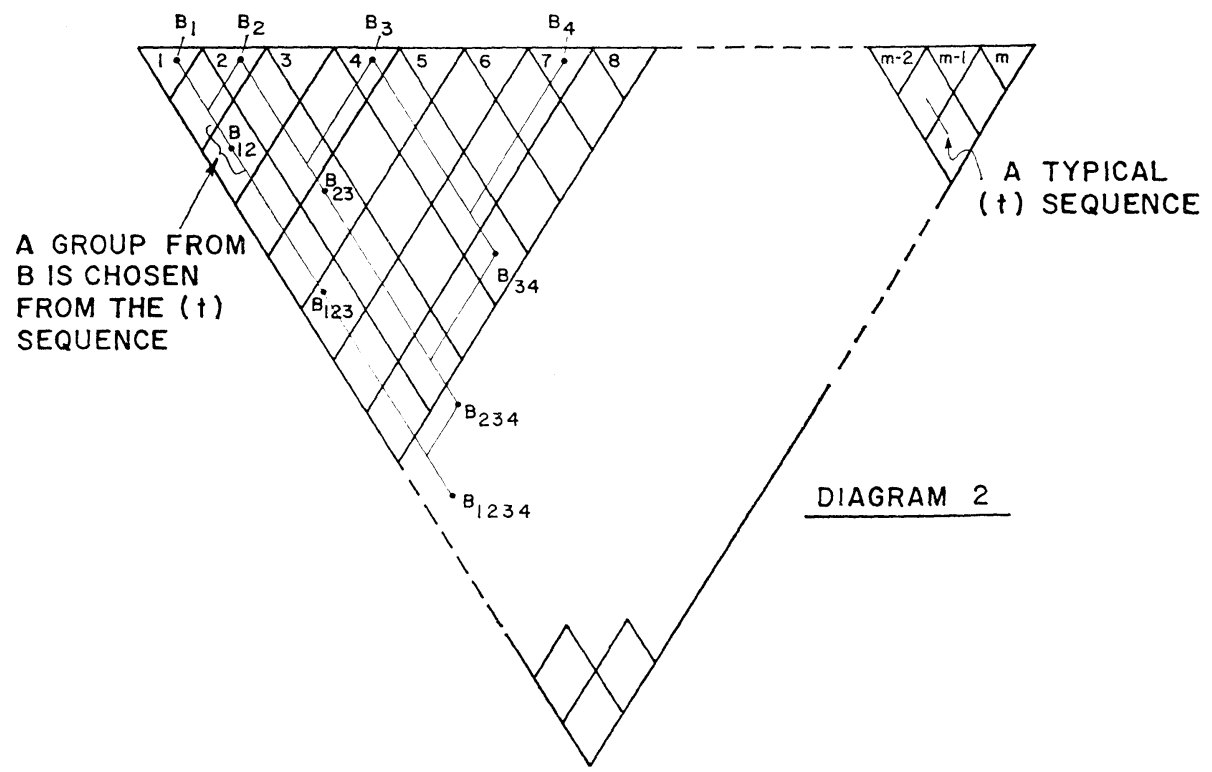

Choose from triangle 1 any group $B_{1}$ from $B$. Say $B_{1}=G_{i, \cdots, i+j}$ where $2 \leqq i \leqq m^{m-1}$ and $0 \leqq j \leqq m^{m-1}-i$. If such a $B_{1}$ does not exist, we are done, for triangle 1 is then an $A$-subtriangle of size $m^{m-1} \geqq n$. Similarly choose from triangle 2 a group $B_{2}=G_{i^{\prime}, \cdots, i^{\prime}+j^{\prime}}$ from $B$, where $m^{m-1}<i^{\prime} \leqq 2 m^{m-1}, 0 \leqq j^{\prime} \leqq 2 m^{m-1}-i^{\prime}$. Now choose a group $B_{12}$ from $B$ from the sequence $G_{i, i+1, \cdots, i^{\prime}+j^{\prime}}, \cdots, G_{i, i+1, \cdots, i^{\prime}+j^{\prime}+m^{m-1}-1}$. (See Diagram 2.) This is a $(+)$ sequence unless $i^{\prime}+j^{\prime}-i<n$. In this case replace $i^{\prime}+j^{\prime}$ with $i+n$ in the above sequence. Now let $B_{3}$ be any group from $B$ found in triangle 4 . The group $B_{23}$ is obtained from the $(+)$ sequence which begins where the diagonals from $B_{2}$ and $B_{3}$ intersect (see Diagram 2). The group $B_{123}$ is obtained 
from the $(+)$ sequence which begins where the diagonals from $B_{12}$ and $B_{23}$ intersect. The group $B_{4}$ is then chosen from triangle 7 and the process continued. Since each new group requires a new $(+)$ sequence, it is not difficult to see that $n(n+1) / 2(+)$ sequences are needed in this process. Hence the requirement $m \geqq n(n+1) / 2$. Thus a $B$-triangle of size $n$ can be constructed and the proof is complete.

REMARK. The number $f(n)=\max \{3 n-1, n(n+1) / 2\}$ is probably much too large as is the triangle size $m^{m}$ used in the proof. These in fact could be lowered slightly, but are retained in the interest of a less complicated proof.

4. Applications. In this section, the results of the previous sections are consolidated to give some information on when completely decomposable groups are radical. We begin with

Definition 4.1. Let $A=\bigoplus_{i \in I} A_{i}$ be any completely decomposable group, and assume $T\left(A_{i}\right)$ is nil for all $i \in I$. Let $\Delta(A)=\sup \{n \mid \exists$ a triangle of size $n$ chosen, possibly with repetitions, from $\left.\left\{A_{i} \mid i \in I\right\}\right\}$. Let $\Delta^{\prime}(A)=\sup \{n \mid \exists$ a triangle of size $n$ chosen without repetition from $\left.\left\{A_{i} \mid i \in I\right\}\right\}$.

Theorem 4.1. Let $A=\bigoplus_{i \in I} A_{i}$ be as above. If $\left\{T\left(A_{i}\right) \mid i \in I\right\}$ is an ordered set and $\Delta(A)$ is finite, then $A$ is radical.

Proof. If $A$ is not radical, then for any $n \geqq 0$, there exists a multiplication on $A$ which yields a nonzero product of length greater than $n$. By Theorem 2.1, $\Delta(A)>n$. This contradicts $\Delta(A)$ finite.

A sort of converse to Theorem 4.1 is provided by

THEOREM 4.2. If $A=\bigoplus_{i \in I} A_{i}$ is completely decomposable with $\Delta^{\prime}(A)=\infty$ then $A$ is not radical.

Proof. If $\Delta^{\prime}(A)$ is infinite, we show there exists a multiplication on $A$ which produces nonzero products of arbitrary length. Let $n$ be any positive integer. Since $\Delta^{\prime}(A)$ is infinite, there exists a triangle $T_{1}$ of size $m>n$ consisting of distinct groups from $\left\{A_{i}\right\}$. Now let $k=f(m+1)$ and form a triangle of size $l \geqq k^{k}$ consisting of distinct groups from $\left\{A_{i}\right\}$. By applying Theorem 3.1 to the disjoint collections of groups $T_{1}$ and $\left\{A_{i}\right\} \backslash T_{1}$, we obtain a subtriangle $T_{2}$ of size $m+1$ consisting of distinct groups from $\left\{A_{i}\right\} \backslash T_{1}$. Continuing this process we obtain a disjoint sequence of collections of groups, $T_{1}, T_{2}, T_{3}, \cdots$, such that distinct groups in $T_{j}$ may be used to form a triangle of 
size at least $m+j-1$. Thus by Theorem 2.1 a ring may be defined on the direct sum of the groups in $T_{j}$ which contains a nonzero product of length $m+j-1$. The direct sum of these rings is then non-nilpotent and is a group direct summand of $A$. Therefore $A$ cannot be radical.

The final result concerns the direct sum of two completely decomposable radical groups.

THeOREM 4.3. Let $A=\bigoplus_{i \in I} A_{i}, B=\bigoplus_{j \in J} B_{j}$ be completely decomposable radical groups such that $\Delta(A)=n$ and $\Delta(B)=m$ are finite. Then if $\left\{T\left(A_{\imath}\right) \mid i \in I\right\} \cup\left\{T\left(B_{j}\right) \mid j \in J\right\}$ is an ordered set, $A \oplus B$ is radical. Moreover, for any multiplication on $A \oplus B,(A \oplus B)^{k^{k}}=0$ for $k=\max \{f(n+1), f(m+1)\}$.

Proof. If $A \oplus B$ is not radical, then $\Delta(A \oplus B)$ is infinite by Theorem 4.1. But Theorem 3.1 then implies that either $\Delta(A)$ or $\Delta(B)$ is infinite, a contradiction. In fact, if $(A \oplus B)^{k^{k}} \neq(0)$ for some multiplication on $A \oplus B$, then $\Delta(A \oplus B) \geqq k^{k}$. But by Theorem 3.1, this implies that $\Delta(A) \geqq n+1$ or $\Delta(B) \geqq m+1$, a contradiction.

\section{REFERENCES}

1. R. A. Beaumont and R. S. Pierce, Torsion free rings, Illinois J. Math., 5, 61-98.

2. L. Fuchs, Abelian Groups, Pergamon Press, New York, 1960.

3. R. Ree and R. J. Wisner, A note on torsion free nil groups, Amer. Math. Soc. Proc., 7, 6-8.

4. T. Szele, Gruppen theorische beziehungen bei gewissen ringkonstruktionen, Math.

Z., 54, 168-180.

5. —, Zur theorie der zeroringe, Math. Ann., 121, 242-246.

6. W. J. Wickless, Abelian groups which admit only nilpotent multiplications, Pacific.

J. Math.,, 40, 251-259.

Received February 5, 1973 and in revised form January 30, 1974.

UNIVERSITY OF CONNECTICUT 


\section{PACIFIC JOURNAL OF MATHEMATICS}

\section{EDITORS}

RICHARD ARENS (Managing Editor)

University of California

Los Angeles, California 90024

R. A. Beaumont

University of Washington

Seattle, Washington 98105
J. DugundJI

Department of Mathematics

University of Southern California

Los Angeles, California 90007

D. Gilbarg and J. Milgram

Stanford University

Stanford, California 94305

\section{ASSOCIATE EDITORS}

E. F. BECKENBACH

B. H. NeUMANN

F. WOLF

K. YosHIDA

\section{SUPPORTING INSTITUTIONS}

UNIVERSITY OF BRITISH COLUMBIA

CALIFORNIA INSTITUTE OF TECHNOLOGY

UNIVERSITY OF CALIFORNIA

MONTANA STATE UNIVERSITY

UNIVERSITY OF NEVADA

NEW MEXICO STATE UNIVERSITY

OREGON STATE UNIVERSITY

UNIVERSITY OF OREGON

OSAKA UNIVERSITY
UNIVERSITY OF SOUTHERN CALIFORNIA

STANFORD UNIVERSITY

UNIVERSITY OF TOKYO

UNIVERSITY OF UTAH

WASHINGTON STATE UNIVERSITY

UNIVERSITY OF WASHINGTON

$\stackrel{*}{*} \stackrel{*}{*} \stackrel{*}{*}$ AMERICAN MATHEMATICAL SOCIETY




\section{Pacific Journal of Mathematics}

\section{Vol. 53, No. $1 \quad$ March, 1974}

Martin Bartelt, Strongly unique best approximates to a function on a set, and a finite

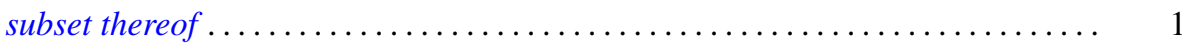

S. J. Bernau, Theorems of Korovkin type for $L_{p}$-spaces $\ldots \ldots \ldots \ldots \ldots \ldots \ldots \ldots \ldots$

S. J. Bernau and Howard E. Lacey, The range of a contractive projection on an

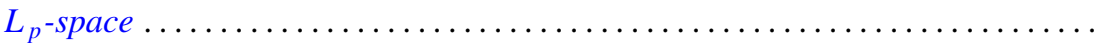

Marilyn Breen, Decomposition theorems for 3-convex subsets of the plane ......... Ronald Elroy Bruck, Jr., A common fixed point theorem for a commuting family of

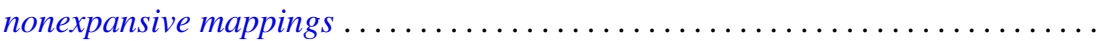

Aiden A. Bruen and J. C. Fisher, Blocking sets and complete $k$-arcs . . . . . . . 73

R. Creighton Buck, Approximation properties of vector valued functions . ......... 85

Mary Rodriguez Embry and Marvin Rosenblum, Spectra, tensor products, and

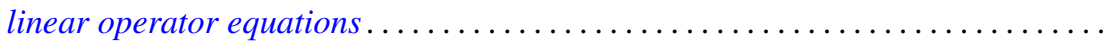

Edward William Formanek, Maximal quotient rings of group rings . . . . . . . . . 109

Barry J. Gardner, Some aspects of T-nilpotence . . . . . . . . . . . . . . . 117

Juan A. Gatica and William A. Kirk, A fixed point theorem for $k$-set-contractions

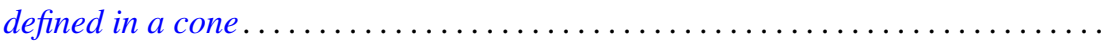

Kenneth R. Goodearl, Localization and splitting in hereditary noetherian prime

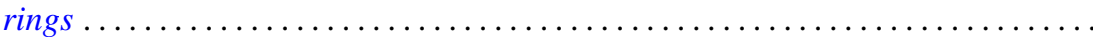

James Victor Herod, Generators for evolution systems with quasi continuous

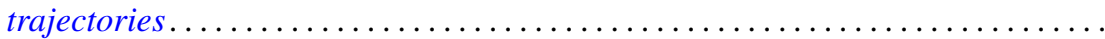

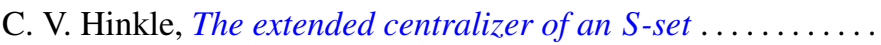

I. Martin (Irving) Isaacs, Lifting Brauer characters of p-solvable groups . . .

Bruce R. Johnson, Generalized Lerch zeta function ...........

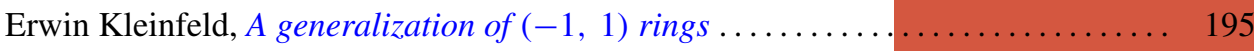

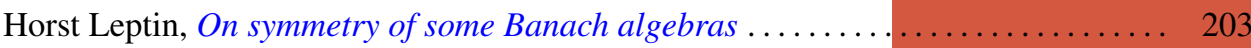

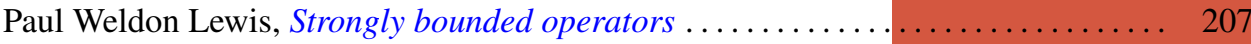

Arthur Larry Lieberman, Spectral distribution of the sum of self-adjoint

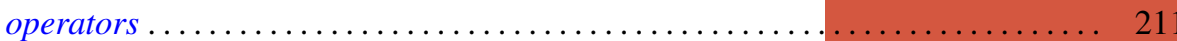

I. J. Maddox and Michael A. L. Willey, Continuous operators on paranormed spaces and matrix transformations

James Dolan Reid, On rings on groups ........................... 229

Richard Miles Schori and James Edward West, Hyperspaces of graphs are Hilbert cubes.

William H. Specht, A factorization theorem for p-constrained groups ...

Robert L Thele, Iterative techniques for approximation of fixed points of certain nonlinear mappings in Banach spaces ...............

Tim Eden Traynor, An elementary proof of the lifting theorem

Charles Irvin Vinsonhaler and William Jennings Wickless, Completely decomposable groups which admit only nilpotent multiplications .

Raymond O’Neil Wells, Jr, Comparison of de Rham and Dolbeault cohomology for

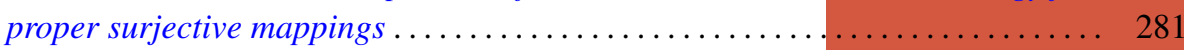

David Lee Wright, The non-minimality of induced central representations . . . . . 301 